\title{
CALIDAD DE LA GESTIÓN ADMINISTRATIVA DEL DIRECTOR Y COMPROMISO LABORAL DE LOS DOCENTES DE ALGUNOS COLEGIOS PRIVADOS SALVADOREÑOS
}

\section{THE QUALITY OF THE PRINCIPAL'S ADMINISTRATIVE MANAGEMENT AND TEACHERS' WORK COMMITMENT IN CERTAIN SALVADORAN PRIVATE SCHOOLS}

\author{
Vinnett Esther Centeno Pérez \\ Departamento de Educación, Asociación Salvadoreña de la IASD, El Salvador \\ vinnette@hotmail.com
}

\section{RESUMEN}

Mediante un estudio descriptivo y correlacional, se analizó la relación entre la percepción de la calidad de la gestión administrativa del director y el grado de compromiso laboral de los docentes de algunos colegios privados de El Salvador, en un contexto donde cada año los docentes renuncian a sus trabajos y pasan a laborar al sector gubernamental. Se administraron dos instrumentos, uno para medir la percepción de la gestión administrativa del director y otro para medir el grado de compromiso de los docentes, a 87 docentes de los siete colegios de las regiones metropolitana y occidental de El Salvador. Se encontró que los docentes tienen un compromiso laboral muy bueno. De igual manera, la percepción que tienen los docentes de la gestión administrativa del director es muy buena. Al observarse la correlación entre ambas variables, se determinó que es positiva y alta. Se observó que cuánto mejor perciben los docentes la gestión administrativa del director mayor es el compromiso con su trabajo. El liderazgo del director es importante para el compromiso laboral de los docentes, por lo cual su selección y nombramiento deben ser realizados con cuidadoso análisis.

Palabras clave: calidad de la gestión administrativa, dirección escolar, compromiso laboral docente

\section{ABSTRACT}

Through a descriptive and correlational study, the relationship between the perception of the quality of the principal's administrative management and the degree of the teachers' work commitment of certain private schools in El Salvador was analyzed, in the context of teachers resigning each year from their jobs and opting to work in the government sector. Two instruments were administered to 87 teachers from the seven schools in the metropolitan and western regions of El Salvador; one to measure the perception of the administrative management of the principal, and another to measure 


\section{CENTENO PÉREZ}

the degree of commitment of teachers. It was found that teachers had an outstanding work commitment. Similarly, the teachers' perception of the principal's administrative management was very good. When the correlation between both variables was observed, it was determined to be positive and high. It was observed that the better the teachers perceived the administrative management of the principal, the higher the commitment they had to their work. The principal's leadership is essential for the teachers' work commitment; thus a principal's selection and appointment must be made with careful analysis.

Keywords: quality of administrative management, school principals, teachers' work committment

\section{Introducción}

Este estudio pretende analizar la percepción de los docentes de algunos colegios privados, ubicados en las áreas geográficas occidental y central de El Salvador, sobre la forma como realizan las gestiones administrativas sus directores. Igualmente, procura encontrar cuál es el grado de compromiso laboral que los docentes tienen hacia el sistema en el que se desempeñan.

\section{Compromiso laboral}

El compromiso laboral se refiere al grado en que un empleado se identifica con una organización específica y con sus metas, además de su deseo por quedarse en ella como integrante. Lai, Luen, Chai y Ling (2014) observaron que los diferentes estilos de liderazgo están relacionados con las tres dimensiones del compromiso laboral (afectivo, continuo y normativo) en lo que se refiere a ambientes administrativos escolares y académicos, donde el estilo de liderazgo de los directores de una institución educativa influirá en el compromiso laboral de los docentes. Un liderazgo que contribuya a la habilidad de generar sinergia y entusiasmo, capaz de enfrentarse a cualquier obstáculo, que cultive la creatividad y sea colaborativo, es un impor- tante predictor del compromiso laboral del docente. Un director con este estilo de liderazgo, que trate individualmente e inspire, motivará a sus subordinados. Los directores que muestren interés en la vida laboral de sus docentes a su vez se sentirán más cercanos emocionalmente y con un fuerte sentido de lealtad y responsabilidad con sus escuelas. Lai et al. (2014) declaran que "esto es bueno para la eficacia y el rendimiento escolar" ( $p$. 73).

\section{Lealtad}

Kumar y Shekhar (2012) mencionan que la lealtad de los empleados a una organización se ha vuelto cada día más importante en este mundo cambiante. Las rutinas de trabajo monótonas, los altos niveles de estrés y estilos dictatoriales de dirigir son factores que ponen en riesgo la lealtad en la organización, lo cual hace que las empresas se esfuercen en establecer buenas relaciones interpersonales con sus empleados y así generen beneficios para la organización. Los empleados constituyen el recurso de inversión más importante para una empresa y hay que cuidarlos.

Zamora Poblete (2009) investigó a docentes de Chile y encontró que los profesores, al sentirse identificados con 
la institución y sentirse acogidos como una familia, manifiestan lealtad. Al involucrarse los docentes con su institución, se apegan a ella y deciden permanecer trabajando por más tiempo en las escuelas, sin importar la edad ni el factor económico. Este estudio muestra que el sentirse identificado con la estructura laboral genera un compromiso afectivo.

Oberholster, Taylor y Cruise (2000) estudiaron el compromiso laboral dentro del sistema de educación que sostiene las escuelas incluidas en este estudio. Ellos relacionaron el compromiso con la madurez de la fe y la antigüedad denominacional del empleado. Estos hallazgos constituyen un antecedente para el presente estudio.

\section{Gestión administrativa}

Las expectativas en lo que respecta a la gestión administrativa en la sociedad actual son cada vez más exigentes a la hora de ejercer un liderazgo direccional. Es evidente que en el ámbito educativo se necesitan líderes efectivos que sean proactivos en sus posturas para mejorar los resultados en satisfacción laboral de sus docentes, en su desarrollo y compromiso (Alam, 2017). Por lo tanto, es necesario revisar los enfoques de liderazgo tradicionales y alinearlos para que sean más productivos.

Para que un director de escuela se enfoque en cuál es la función que le corresponde hacer, es importante que sepa la diferencia entre las funciones de manejo de una institución y las funciones de administrar. Entre las funciones de manejo en una organización están las siguientes: planear, organizar, apoyar, dirigir y controlar. Las funciones para administrar incluyen asistencia a reuniones, registros, disposición de materiales y libros de texto, apoyo en los currícu- los de instrucción y enseñanza, comunicación e implementación de materiales (Samkange, 2013).

En un estudio realizado por Okutan (2014) con directores de escuelas en Turquía, se analizó el tipo de gestión administrativa que mostraban en su liderazgo. Entre los hallazgos relevantes, se observó el estilo clásico inadecuado de liderazgo que presentó deficiencias para crear un clima positivo en la enseñanza-aprendizaje. Los directores solo estaban preocupados por mantener su estatus quo aplicando los reglamentos y las leyes, sin tomar iniciativas. En sus habilidades de comunicación demostraron ser incompetentes, de modo que no motivaban a sus docentes, ni eran justos o imparciales. Quedó demostrado que no son suficientes los conocimientos básicos y se les recomendó participar de un programa de educación continua para mejorar sus habilidades. Un director que no se involucra con su entorno ni con sus maestros, que no es imparcial, no motiva ni aprovecha los nuevos enfoques de la educación, ni contribuye significativamente a la institución que dirige. Por lo tanto, de aquí se deriva el principio de que es muy importante y necesario que un director esté continuamente involucrado en un aprendizaje significativo, para realizar una gestión administrativa innovadora en su organización.

\section{Características personales}

Wasserman, Ben-Eli, Yehoshua y Gal (2016) mencionan que el rol del director es influenciado por sus valores personales y su percepción mental. Cuánto más descentralizado sea el estilo de la gestión del director, mayor será la percepción positiva del profesor. Los estudios han demostrado que la participación de los docentes y el fomento de esta 
participación en la toma de decisiones constituyen un medio para desarrollar la escuela como un todo y para mejorar la enseñanza.

No hay duda de que el avance profesional, la aceptación de roles, la independencia y la participación del maestro en la toma de decisiones aumentan su satisfacción y alientan su persistencia en la enseñanza. Wasserman et al. (2016) encontraron que "cuanto más un maestro esté dedicado activamente a la escuela más dispuesto estará en dedicar su tiempo y energía a sus actividades escolares" (p. 189).

\section{Toma de decisiones}

Según Alam (2017), el director debe comprender que bajo su rol motiva, dirige y evalúa el desempeño de los empleados. Wachira, Gitumu y Mbugua (2017) mencionaron que muchas veces el no involucrar a los docentes en la toma de decisiones hace que no sea efectivo el liderazgo escolar. En la mayoría de las escuelas donde hay una pobre participación de los docentes disminuyen los logros. Es aquí donde el director establece el ritmo que guiará y motivará tanto a los docentes como a sus alumnos. También el comportamiento en el liderazgo del director impactará significativamente la moral del docente y el logro de los estudiantes, en beneficio de la reducción de los problemas de disciplina.

Un estudio realizado por Saad (2012) observó que, al participar en la toma de decisiones, los maestros tuvieron un alto nivel de compromiso laboral. Al involucrarse y estar incluidos en decisiones de su institución, toman interés en ella y se comprometen en sus labores. Una gestión administrativa que involucra al personal docente en un diálogo abierto donde se tomen decisiones consensua- das es un factor motivante en el compromiso laboral.

Definitivamente, el tipo de liderazgo que ejerza el director de una escuela es determinante para que los demás lo sigan y sientan que hay dirección en lo que se realiza. En un estudio realizado por Ward (2013), los docentes, al participar de la visión y de la misión de la institución en la que trabajaban, se sintieron comprometidos como equipo en sacar adelante a su institución. Dentro del ambiente escolar es necesario que todos sus segmentos, maestros, alumnos, director y el sistema escolar distrital, se conjuguen en favor de sacar adelante la organización donde se propicie una buena cultura escolar. Como dicen Leithwood y Riehl (citados en Clayton, 2014), "es importante lo que haces, pero es más importante cómo lo haces dependiendo del ambiente y las circunstancias" (p. 73). El liderazgo educativo que exista en determinada institución deberá tener una mezcla de la capacidad del que dirige y del compromiso, habilidades y capacidad de los docentes.

\section{Clima organizacional}

Al estudiar el comportamiento de los empleados, García Rivera, Mendoza Martínez y Puerta Sierra (2012) encontraron que un ambiente de inseguridad e inestabilidad laboral genera una relación negativa con relación el compromiso laboral. Calik, Sezgin, Kavgaci y Kilinc (2012) descubrieron que la respuesta de los docentes en términos de su eficiencia personal y de su eficiencia colectiva depende del liderazgo educativo. En otras palabras, según sea su percepción del liderazgo, aumentará o disminuirá su compromiso con sus labores. Cuán importante es contar con un líder educativo que reconozca que su gestión contribuye 
a mejorar el clima organizacional, o, por otro lado, a bajar el rendimiento de sus empleados. Al sentirse comprometido en mejorar su gestión, indudablemente contribuirá al bienestar del clima organizacional.

Cuando los empleados sienten que existe un trato justo, de respeto, esto conlleva a tener una lealtad más con su trabajo y con sus jefes (Jiang y Cheng, 2008). Las personas perciben si son tratadas con dignidad y ética y son valoradas. Es importante gozar de buenas relaciones interpersonales en un ambiente laboral. Esto establecerá vínculos más fuertes entre el grupo, no importando la jerarquía.

Según Restrepo-Abondano y Restrepo-Torres (2012), el director de una institución debe ser una persona que guía y practica diariamente un liderazgo adaptativo. Su vida es una enseñanza. Da equilibrio y tiene una postura centrada para aplicar los reglamentos y manuales. Es una figura clave en el proceso de cambio. Proporciona confianza y está interesado en un ambiente de estudio con altos estándares de calidad. Promueve un ambiente de trabajo positivo. También desarrolla habilidades para buscar la manera de sostener los recursos de la institución que dirige. Está comprometido con las familias de los estudiantes y con la sociedad. Inspira nuevas visiones y enfoques educativos. Está a la vanguardia en las diferentes áreas y desafíos de su institución. Estas son las expectativas del liderazgo adaptativo, comprometido con los procesos de aprendizaje, el sistema cultural y tecnológico donde se satisfacen las demandas del entorno.

\section{Flexibilidad}

Watkinks y Moak (citados en Mack, 2016) mencionan que, cuando el direc- tor reconoce los componentes del conocimiento que construyen una buena relación entre los maestros y los estudiantes, se pueden desarrollar estrategias eficientes de servicio al cliente y de educación de calidad, dos áreas a las que apuesta para conseguir más estudiantes y que salgan bien preparados.

Como en todo círculo de trabajo siempre hay situaciones personales que muchas veces interfieren con las cargas del quehacer, hay una lista de asuntos que el director debería tomar en cuenta a la hora de su gestión administrativa:

1. Tomar tiempo para conversar informalmente con sus maestros. Mejorar sus relaciones en términos de lenguaje corporal y de lenguaje verbal, que siempre debe ser positivo.

2. Permitir que los docentes den sus opiniones sobre el funcionamiento de la institución y que se familiaricen con las decisiones.

3. Respetar los sentimientos y las necesidades individuales de los maestros y brindarles apoyo de manera concreta. Brindar confianza.

4. Mostrar un carácter y un comportamiento moralmente aceptables y consistentes con sus creencias y valores.

\section{Metodología \\ Tipo de investigación}

Este estudio fue descriptivo y correlacional.

\section{Participantes}

La población estuvo formada por 91 docentes de siete colegios del sistema educativo de las asociaciones Occidental Salvadoreña y Metropolitana Salvadoreña de la Iglesia Adventista. Ellos laboraban en los niveles que van desde la educación parvularia hasta el bachillerato (ver Tabla 1). No hubo 
muestreo, sino que se censó la totalidad de la población.

Tabla 1

Distribución de los docentes de la muestra por colegios

\begin{tabular}{|c|c|}
\hline Colegio & $\mathrm{n}$ \\
\hline Ahuachapán & 4 \\
\hline Santa Ana & 17 \\
\hline Sonsonate & 18 \\
\hline El Zarzal & 7 \\
\hline Central San Salvador & 14 \\
\hline Las Margaritas & 13 \\
\hline Scandia & 18 \\
\hline Total & 91 \\
\hline
\end{tabular}

\section{Instrumentos de medición}

Para esta investigación se utilizaron dos instrumentos. El instrumento para evaluar el compromiso laboral fue elaborado por Méndez Cruz y Meza Escobar (Méndez Cruz, 2015) e incluye 19 ítems. Este instrumento fue utilizado por Ontiveros Ramírez (2016) entre los maestros de enseñanza media de la Unión Mexicana del Norte, donde obtuvo un coeficiente de confiabilidad alfa de 936 .

El instrumento que mide la variable de gestión administrativa en el director fue diseñado por del Va1le López y Meza Escobar (del Valle López, 2016). Este instrumento fue adaptado para ser aplicado a la población en estudio y consta de 21 ítems. Fue utilizado además por González de la Rosa (2016) entre los maestros de enseñanza media de los colegios adventistas de República Dominicana, donde obtuvo un coeficiente de confiabilidad alfa de .972 .

Variables. Este estudio tuvo como variable independiente la percepción de la calidad de la gestión administrati- va del director de cada uno de los siete colegios participantes. Esta variable utilizó una escala Likert de siete puntos: pésimo(a) (1), muy malo(a) (2), malo(a) (3), regular (4), bueno(a) (5), muy bueno(a) (6) y excelente (7). Como variable dependiente, se midió el compromiso laboral de los docentes participantes. Esta variable utilizó una escala Likert de siete puntos: totalmente en desacuerdo (1), muy en desacuerdo (2), algo en desacuerdo (3), indeciso (4), algo de acuerdo (5), muy de acuerdo (6) y totalmente de acuerdo (7).

\section{Resultados \\ Caracterización de la muestra}

Participaron en la investigación los docentes de los siete colegios adventistas de las Asociaciones Occidental y Metropolitana Salvadoreñas. Las encuestas se aplicaron en 2019 a un total de 87 docentes.

Género. En la Tabla 2 se muestra la distribución de los maestros encuestados de acuerdo con su género. Como puede observarse, el mayor número de participantes correspondió al género femenino. Dos de ellos no indicaron su género.

Tabla 2

Distribución de los participantes por género

\begin{tabular}{lcc}
\hline \multicolumn{1}{r}{ Género } & $n$ & $\%$ \\
\hline Masculino & 28 & 32.9 \\
Femenino & 57 & 67.1 \\
Total & 85 & 100.0 \\
\hline
\end{tabular}

Estado civil. En la Tabla 3 se muestra la distribución de los maestros encuestados de acuerdo con su estado civil. Como puede observarse, el mayor número de los participantes fue el de los casados. 
Tabla 3

Distribución de los participantes por estado civil

\begin{tabular}{|c|c|c|}
\hline Estado civil & $n$ & $\%$ \\
\hline Soltero & 28 & 32.2 \\
\hline Casado & 58 & 66.7 \\
\hline Otro & 1 & 1.1 \\
\hline Total & 87 & 100.1 \\
\hline
\end{tabular}

Categoría salarial. En la Tabla 4 se observa la distribución de los participantes del estudio según su categoría salarial. Cuatro de ellos no especificaron su categoría salarial.

Tabla 4

Distribución de los participantes por categoría salarial

\begin{tabular}{lcc}
\hline \multicolumn{1}{c}{ Categoría salarial } & $n$ & $\%$ \\
\hline Por contrato & 44 & 53. \\
Regular & 39 & 47.0 \\
Total & 83 & 100.0 \\
\hline
\end{tabular}

Años de servicio. En la Tabla 5 se observa la distribución de los participantes del estudio según el rango de sus años de servicio. Dos de ellos no especificaron el rango de sus años de servicio.

Tabla 5

Distribución de los participantes por años de servicio

\begin{tabular}{lcc}
\hline \multicolumn{1}{r}{ Años de servicio } & $n$ & $\%$ \\
\hline Hasta 10 años & 49 & 57.6 \\
11 a 20 años & 20 & 23.5 \\
21 a 30 años & 13 & 15.3 \\
31 años o más & 3 & 3.5 \\
Total & 85 & 100.0 \\
\hline
\end{tabular}

\section{Confiabilidad de los instrumentos}

Para poder calcular la confiabilidad del instrumento, se utilizó el índice alfa de Cronbach. Para el instrumento de compromiso laboral en el procedimiento de casos se tomó como válido el $92 \%$, excluyendo el $8 \%$ de los casos. El valor alfa de Cronbach que se obtuvo para los 19 ítems del instrumento fue de .872 . Según este resultado, se considera que es un instrumento confiable.

Para el instrumento de gestión administrativa, en el procesamiento de casos, se tomó como válido el $89.7 \%$ y se excluyó el $9 \%$ de los casos. El valor alfa de Cronbach que se obtuvo para los 21 ítems del instrumento fue de .983 . Este resultado muestra que dicho instrumento es confiable.

\section{Comportamiento de las variables de estudio}

Para medir la variable de compromiso laboral, cada uno de los 19 ítems se midió con una escala Likert que va desde un valor totalmente en desacuerdo (1) hasta totalmente de acuerdo (7), observándose un rango de valores obtenido entre 19 y 133 puntos.

Para los 87 participantes, se obtuvo una media aritmética de 6.18 y una desviación estándar de .658. Según la escala de medición que se utilizó, la media aritmética encontrada indica que los maestros tienen un compromiso laboral muy bueno.

Los indicadores que obtuvieron mayor valor, representado por su media aritmética, fueron los siguientes: (a) "Defiendo esta institución cuando hablan injustamente de ella" ( $M=6.82$, $D E=.467)$, (b) "Soy feliz con el trabajo que realizo" ( $M=6.78, D E=.538) \mathrm{y}$ (c) "Me siento incómodo(a) cuando no realizo bien mi trabajo" $(M=6.75, D E$ $=.463$ ).

Los indicadores que tuvieron menor valor, representado por su media 
aritmética, fueron los siguientes: (a) "Suplo todas mis necesidades económicas con este trabajo" $(M=4.85, D E$ $=1.74$ ), (b) "Dejar esta institución representa una pérdida económica" $(M=$ $5.01, D E=1.64)$ y (c) "Dispongo de todos los recursos necesarios para realizar mi trabajo" $(M=5.38, D E=1.50)$.

Para medir la variable gestión administrativa, cada uno de los 21 ítems se midió con una escala Likert que va desde pésimo (1) hasta excelente (7). Se observó un rango de valores obtenido entre 21 y 147 puntos.

Para los 87 participantes, se obtuvo una media aritmética de 6.05 y una desviación estándar de 1.20. Según la escala de medición que se utilizó, la media aritmética encontrada indica que los maestros tienen una percepción muy buena de la gestión administrativa del director.

Los indicadores que obtuvieron mayor valor, representado por su media aritmética, fueron los siguientes: (a) "Actuar de acuerdo a principios cristianos" $(M=6.43, D E=1.16)$, (b) "Mantener buenas relaciones con los empleados" $(M=6.30, D E=1.39)$ y (c) "Tratar en privado asuntos que requieren disciplina para un empleado" $(M=6.30, D E$ $=1.44$ ).

Los indicadores que obtuvieron menor valor, representado por su media aritmética, fueron los siguientes: (a) "Distribuir equitativamente las asignaciones laborales entre los empleados" $(M=5.84, D E=1.60)$, (b) "Planear con eficacia" ( $M=5.84, D E=1.47)$ y (c) "Planificar con creatividad" $(M=5.85$, $D E=1.53)$.

\section{Análisis correlacional}

El objetivo de la investigación es determinar si la gestión administrativa del director percibida por los docentes se relaciona significativamente con su compromiso laboral. Para ello, se utilizó la prueba estadística $r$ de Pearson. Las variables consideradas fueron la percepción de la gestión administrativa del director y el grado de compromiso laboral.

Al correr la prueba estadística, se encontró un valor $r$ de .682 y un nivel de significación $p$ de .000 . Para dicho nivel de significación, se consideró que la relación entre las variables es positiva y alta. La percepción de la gestión administrativa explica el $46.5 \%$ de la varianza del compromiso laboral.

\section{Otros hallazgos}

Se realizaron otras pruebas estadísticas para tratar de encontrar alguna diferencia significativa de las puntuaciones de las variables en estudio - compromiso laboral y gestión administrativa percibida - entre los grupos determinados por las diferentes variables demográficas.

Género. Se procuró determinar algunas diferencias significativas de las puntuaciones de las variables del estudio entre los grupos definidos por la variable género. No se encontró diferencia significativa entre mujeres y hombres en el compromiso laboral $\left(t\left(_{83}\right)=-1.502, p=\right.$ .137) ni en la gestión administrativa percibida $\left.\left(t_{83}\right)=-1.516, p=.133\right)$.

Estado civil. Se encontró que existe una diferencia ligera pero significativa entre los solteros y casados $\left(t\left(_{84}\right)=\right.$ $-2.242, p=.028)$. Los casados manifiestan un compromiso laboral significativamente más alto $(M=6.290)$ que los solteros $(M=5.956)$. No se observaron diferencias significativas de percepción de la gestión administrativa entre solteros y casados $\left(t\left(_{84}\right)=-1.614\right.$, $p=.110)$. 
Al comparar el nivel de correlación entre ambas variables principales del estudio, se observó que, en el grupo de casados, la correlación es más alta $(r=$ $.723, p=.000)$ que en el de los solteros $(r=.577, p=.001)$.

Categoría salarial. Entre los grupos definidos por su categoría salarial, no se observó diferencia significativa en la percepción de la gestión administrativa $\left(t\left(_{81}\right)=.786, p=.434\right)$ ni en su compromiso laboral $\left(t\left(_{81}\right)=-1.594, p=.054\right)$ pero sí una tendencia en la variable de compromiso laboral, ya que los docentes regulares mostraron un compromiso mayor $(M=6.345)$ que los docentes de contrato $(M=6.074)$.

Al comparar el nivel de correlación entre ambas variables principales del estudio, se observó que, en el grupo de docentes regulares, la correlación es más alta $(r=.810, p=.000)$ que en el de los docentes por contrato $(r=.654$, $p=.000$ ).

Años de servicio. Para el análisis de la variable años de servicio, se conformaron dos grupos (hasta 10 años y 21 o más años). No se observó diferencia significativa entre los grupos en su percepción de la gestión administrativa $\left(t_{63}\right)=-.293, p$ $=.770$ ), pero se observó una diferencia significativa, aunque ligera, en su compromiso laboral $\left(t\left(_{63}\right)=-2.082, p=.041\right)$. El grupo de docentes con mayor antigüedad laboral mostró una media $(M=$ 6.457) más alta que la de los docentes con menor antigüedad laboral $(M=6.050)$.

Igualmente al comparar el nivel de correlación entre ambas variables principales del estudio, se observó que, en el grupo de docentes de 21 o más años de antigüedad laboral, la correlación es mucho más alta $(r=.953, p=.000)$ que en el de los docentes con una antigüedad laboral de hasta 10 años $(r=.732, p=.000)$.

\section{Discusión}

Al analizar los resultados de esta investigación, se encontró que el grado de calidad de la gestión administrativa del director percibida por los docentes está significativamente relacionada con su compromiso laboral. La gestión que realiza diariamente un director está correlacionada con la forma como sus docentes se desempeñarán en la institución, como Lai et al. (2014) lo confirmaron en su estudio sobre los ambientes administrativos escolares. Sin lugar a duda, los directores influyen en el compromiso laboral. Respaldan esta idea Shaw y Newton (2014), quienes observaron que la actitud del director en la gestión directiva es de vital importancia, al demostrar interés en la forma como se relaciona con sus docentes. Esto beneficia a su satisfacción y al grado de su compromiso laboral.

Se analizó el estado civil en su relación con el compromiso laboral y se encontró que los casados manifestaron estar más interesados que los solteros en su trabajo. Esto podría deberse a que algunos casados tienen diversos compromisos, entre los cuales se destaca principalmente el financiero. Es probable que los solteros estén buscando oportunidades de desarrollo y crecimiento personal, por lo cual están más vulnerables en relación al compromiso laboral. Este resultado coincide con el de García Rivera et al. (2012), quienes encontraron que los ambientes de inseguridad e inestabilidad laboral están negativamente correlacionados con el compromiso laboral. De igual manera, si no perciben oportunidades de crecimiento laboral, es probable que los solteros decidan salir del sistema educativo adventista.

Entre los resultados, se encontró que los docentes regulares, quienes tienen 
mejor estabilidad salarial, mostraron un compromiso mayor en el aspecto laboral. Kumar y Shekhar (2012) sostienen que los empleados constituyen el recurso de inversión más importante para una empresa. Para contar con empleados leales, se necesita ofrecerles más que un trabajo. Hay que darles compensaciones apropiadas, invertir en su formación y desarrollo y darles reconocimiento y premiaciones, entre otros elementos. Por eso es importante buscar la manera de lograr que los docentes de contrato sean regularizados.

En esta investigación, se encontró que los docentes con mayor antigüedad, de 21 años o más, están más comprometidos laboralmente. Este hallazgo coincide con el de Oberholster et al. (2000), quienes relacionaron un alto compromiso del empleado con su antigüedad en el empleo. Este resultado podría atribuirse al hecho de que los docentes de mayor antigüedad sobrepasaron tiempos difíciles, incluyendo problemas económicos, pero están aún dentro del sistema educativo adventista, demostrando lealtad a su denominación, entrega al ministerio de la enseñanza que realizan en los colegios, amor a sus estudiantes y satisfacción por ser facilitadores de los cambios en sus alumnos. Estos docentes aman lo que hacen y están dispuestos a jubilarse dentro del sistema. Entre el grupo de 16 docentes con más de 20 años de antigüedad, se observó que 12 de ellos (75\%) pertenece al género femenino. En el estudio que realizaron Chiang Vega, Nuñez Partido, Martín y Salazar Botello (2010), "las mujeres están presionadas más por el deber y los hombres, si se sienten reconocidos, aumentan su compromiso afectivo con la organización" (p. 99). La permanencia en el sistema de estas docentes posiblemente se deba al hecho de ser madres y sacar adelante sus responsabilidades en el hogar.

$\mathrm{Al}$ analizar la encuesta sobre el compromiso laboral, los docentes asignaron el puntaje más alto a la declaración según la cual ellos defienden la institución cuando hablan injustamente de ella. La segunda declaración que obtuvo mayor puntaje enuncia que son felices con el trabajo que realizan. Como puede observarse, estos datos muestran que tienen un sentido de pertenencia a la institución. Tienen agrado por realizar sus tareas en sus instituciones. Esto explica por qué en su percepción de la gestión administrativa del director ellos asignaron mayor puntaje a las declaraciones según las cuales el director "actúa de acuerdo a los principios cristianos" y "mantiene buenas relaciones con sus empleados". En otras palabras, se sienten satisfechos porque están en un ambiente de trabajo cristiano que manifiesta buenas relaciones humanas. Tienen la ventaja de contar con un líder que actúa de acuerdo con los principios cristianos. Y es justamente lo que los colegios adventistas ofrecen a la comunidad, una enseñanza basada en valores y principios cristianos. La sociedad salvadoreña está plagada de acciones violentas que no contribuyen a la formación del carácter de los niños y jóvenes. Está sedienta de instituciones que promuevan un entorno de aprendizaje y que fomenten los buenos principios.

Uno de los ítems de la encuesta compromiso laboral que obtuvo el menor puntaje declara que los docentes no "suplen todas sus necesidades económicas con este trabajo". Es lamentable, pero esta es una de las carencias observadas. No todos los colegios tienen los recursos disponibles para pagar salarios atractivos y brindar las ayudas necesarias al docente. Hacen un esfuerzo sumamente 


\section{CALIDAD DE LA GESTIÓN ADMINISTRATIVA DEL DIRECTOR}

grande y excepcional por pagar los salarios mínimos del país. De igual manera, y relacionado con las finanzas, se puede observar que la declaración "dejar esta institución representa una pérdida económica" ocupa el segundo lugar de menor valor, porque en realidad no pierde nada el docente al abandonar su institución. No representa una opción atractiva laborar en estas instituciones educativas debido a las restricciones financieras. Otra declaración que demuestra las grandes necesidades financieras que atraviesan la mayoría de estos colegios se ve reflejada en la declaración "dispongo de todos los recursos necesarios para realizar mi trabajo", cuyo valor está dentro de los más bajos.

En relación a la gestión administrativa del director percibida por los docentes, la declaración "distribuir equitativamente las asignaciones entre los empleados", es la que obtuvo menor puntaje. Un director que no distribuye equitativamente muestra favoritismo, lo que constituye una deficiencia en el liderazgo del director. Una persona con este defecto de carácter no es bien vista dentro del gremio de maestros. También las declaraciones "planear con eficiencia" y "planificar con creatividad" se hallan entre las que obtuvieron las puntuaciones más bajas. Un director que no es efectivo en su trabajo administrativo puede causar conflictos y problemas en el personal.

\section{Referencias}

Alam, S. (2017). A study on leadership styles executed by principal and academic coordinator in one of the private schools in Gilgit-Baltistan, Pakistan. Academic Research International, 8(3), 71-78.

Calik, T., Sezgin, F., Kavgaci, H. y Kilinc, A. (2012). Examination of relationships between instructional leadership of school principal's and self-efficacy of teacher and collective tea- cher efficacy. Educational Sciences: Theory \& Practice, 12(4), 2469-2504.

Chiang Vega, M., Núñez Partido, A., Martín, M. J. y Salazar Botello, M. (2010). Compromiso del trabajador hacia su organización y la relación con el clima organizacional: un análisis de género y edad. Panorama Socioeconómico, 28(40), 92-103.

Clayton, J. K. (2014). The leadership lens: Perspectives on leadership from school district personnel and university faculty. International Journal of Educational Leadership Preparation, 9(1), 58-75.

Del Valle López, J. (2016). Modelo asociativo entre factores determinantes del desempeño organizacional y la satisfacción de los públicos (Tesis doctoral). Universidad de Montemorelos, Montemorelos, Nuevo León, México.

García Rivera, B. R., Mendoza Martínez, I. A. y Puerta Sierra, L. M. (2012). ¿Es el downsizing un factor de impacto sobre los comportamientos innovadores, el compromiso organizacional y las capacidades de aprendizaje de los trabajadores de una empresa de alimentos en México? Revista Internacional Administración y Finanzas, 5(3), 57-78.

González de la Rosa, J. (2016). Modelo de factores predictores de desempeño e imagen institucional validado en colegios confesionales dominicanos (Tesis doctoral). Universidad de Montemorelos, Montemorelos, Nuevo León, México.

Jiang, D. Y. y Cheng, B. S. (2008). Affect- and role-based loyalty to supervisors in Chinese organizations. Asian Journal of Social Psychology, 11, 214-221. https//doi.org/10.111/j .14-67-839X.2008.00260.x

Kumar, D. N. S. y Shekhar, N. (2012). Perspectives envisaging employee loyalty: A case analysis. Journal of Management Research, 12(2), 110112. https://doi.org/10.2139/ssrn.1961430

Lai, T., Luen, W., Chai, L. y Ling, L. (2014). School principal leadership styles and teacher organizational commitment among performing schools. The Journal of Global Business Management, 20(2), 67-75.

Mack, K. (2016). The perceptions of the leadership behaviors of elementary school principals through professional experience in Texas (Tesis doctoral). University of Phoenix, Phoenix, EE. UU.

Méndez Cruz, A. (2015). Clima y compromiso organizacional percibido por los empleados del parque Eco arqueológico en México (Tesis de maestría). Universidad de Montemorelos, Montemorelos, Nuevo León, México.

Mustapha, N., Zainal Abidin, M. Z. y Saufi, S. (2013). Measuring the influence of dispositional 


\section{CENTENO PÉREZ}

characteristics and motivational factors on employee loyalty among teachers at private Islamic schools in Kelantan, Malaysia. International Review of Social Sciences \& Humanities, 5(2), 127-134.

Oberholster, F. R., Taylor V, J. W. y Cruise, R. J. (2000). Spiritual well-being, faith maturity, and the organizational commitment of faculty in Christian colleges and universities. The Journal of Research on Christian Education, 9(1), 31-60. https://doi.org/ 10.1080/10656210009484896

Okutan, M. (2014). My school principal is not a leader. Education, 135(1), 93-100.

Ontiveros Ramírez, F. (2016). Modelo de asociación entre factores predictores del desempeño y compromiso laboral validado en maestros del corporativo educativo adventista de la Unión Mexicana del Norte (Tesis doctoral). Universidad de Montemorelos, Montemorelos, Nuevo León, México.

Restrepo-Abondano, J. M. y Restrepo-Torres, M. L. (2012). Cinco desafíos en el ejercicio del liderazgo en los rectores de colegios. Educación y Educadores, 15(1), 117-119.

Saad, N. (2012). The effects of teacher's participation in decision making of commitment. The International Journal of Interdisciplinary Social Sciences, 6(9), 1-16.

Samkange, W. (2013). Management and administration in education: What do school heads do?
A focus of primary school heads in one district in Zimbabwe. International Journal of Social Sciences and Education, 3(3), 635-643.

Shaw, J. y Newton J. (2014). Teacher retention and satisfaction with a servant leader as principal. Education, 135(1), 101-106.

Wachira, F. M., Gitumu, M. y Mbugua, Z. (2017). Effect of principal's leadership styles on teachers' job performance in public secondary schools in Kieni West Subcounty. International Journal of Humanities and Social Science Invention, 6(8), 72-86.

Ward, C. J. (2013). Why leadership matters: One school's journey to success. Educational Leadership and Administration Teaching and Program Development, 24, 62-74.

Wasserman, E., Ben-Eli, S., Yehoshua, O. y Gal, R. (2016). Relationship between the principal's leadership style and teacher motivation. International Journal of Learning, Teaching and Educational Research, 15(10), 180-192.

Zamora Poblete, G. (2009). Compromisos organizacionales de los profesores chilenos y su relación con la intención de permanecer en sus escuelas. Revista Latinoamericana de Psicología, 4l(3), 445-460.

Recibido: 28 de febrero de 2019

Revisado: 13 de marzo de 2019 Aceptado: 14 de abril de 2019 